\title{
HEMOGLOBINA GLICOSILADA COMO ELEMENTO PRONÓSTICO EN LAS COMPLICACIONES MACROVASCULARES DE LA DIABETES MELLITUS ${ }^{1}$
}

Agnes Fajardo Matarrita ${ }^{2}$ Silvia Gutiérrez Oviedo ${ }^{3}$

Institución: Hospital de la Anexión, Guanacaste, Costa Rica

\section{COMO CITAR}

Fajardo, A.; Gutiérrez, S.(2012).Hemoglobina Glicosilada como elemento pronóstico en la complicaciones macrovasculares de la Diabetes Mellitus.[En línea].Rev. Enfermería Actual en Costa Rica, 22, 1-9 [citado (fecha)]. Disponible World World Wide Web:

$<$ http://www.revenf.ucr.ac.cr/hemoglobina.pdf> ISSN 1409-4568

\section{RESUMEN}

En este artículo se discute la información obtenida respecto del cuidado enfermero brindado a personas portadoras de Diabetes Mellitus tipo 2 relacionada con la elevación de la hemoglobina glicolisada (Hba1c) como factor pronóstico para el desarrollo de complicaciones. Se realizó una búsqueda de la mejor información científica disponible para construir el estado de la cuestión. Posteriormente, se estableció una pregunta en formato PICO (pacientes, intervención, comparación, observación) y se procedió a establecer estrategias de búsqueda en las diferentes bases de datos sugeridas en el Curso de Práctica Clínica de Enfermería Basada en la Evidencia impartido por CIEBE-CR, entre las que se encuentran PUBMED, EBSCO HOST, principalmente. El análisis crítico se llevó a cabo mediante las recomendaciones que brinda CASPe y se comparó con la práctica realizada en el Hospital de la Anexión. El control intensivo de la glicemia, el cual incluye agentes orales, la insulina y la intervención cardiovascular múltiple reflejada en hemoglobinas glicosilada menores a 7 , indicó que existía una evidente disminución en la incidencia de eventos macrovasculares mayores tales como el infarto agudo al miocardio, el ictus no fatal y el accidente vascular cerebral. De acuerdo con la evidencia, mantener la hemoglobina glicosilada dentro de parámetros normales disminuye el riesgo de sufrir complicaciones macrovasculares de esta patología en comparación con aquellos pacientes que manejan hemoglobina glicosilada alta.

Palabras clave: complicaciones, diabetes mellitus, hemoglobina-glicosilada

\footnotetext{
${ }^{1}$ Fecha de recepción: 16 de noviembre 2011

Fecha de aceptación: 16 de enero 2012

${ }^{2}$ Enfermera especialista en Administración de Servicios de Salud. Coordinadora del Programa Hospital de Día. Hospital de la Anexión, Guanacaste. Correo electrónico: agnesfm2010@hotmail.com

${ }^{3}$ Enfermera Educadora en Servicio. Hospital de la Anexión, Guanacaste. Correo electróncio silgutio@gmail.com
} 


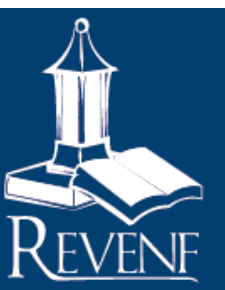

\section{Revista Electrónica Enfermería Actual en costa Rica}

\section{GLYCOSYLATED HAEMOGLOBIN AS A PROGNOSIS MACROVASCULAR COMPLICATIONS OF DIABETES MELLITUS ${ }^{1}$}

Viriam Leiva Díaz ${ }^{2}$ Pilar Acosta Rojas ${ }^{3}$

Institution: Hospital de la Anexion, Guanacaste, Costa Rica

\section{CITED}

Fajardo, A.; Gutiérrez, S. (2012).Glycosylated Haemoglobin as a prognosis macrovascular complications of Diabetes Mellitus.[Electronic version].Rev. Enfermería Actual en Costa Rica, 22, 1-9 [cited (date)]. Available World World Wide Web: $<$ http://www.revenf.ucr.ac.cr/hemoglobina.pdf> ISSN 1409-4568

\section{ABSTRACT}

This article discusses the information obtained with respect to nursing care provided to people living with type 2 diabetes associated with elevated glycosylated hemoglobin (HbA1c) as a prognostic factor for the development of complications. A search of the best scientific information available to approach the state of the matter. Subsequently established a question in PICO format (patient, intervention, comparison, outcome) and proceeded to establish search strategies in different databases suggested in the Course of Clinical Practice Evidence-Based Nursing conducted by CIEBE-CR (Collaborative program for research in evidence based nursing of Costa Rica); we explored daba base as PubMed, EBSCO HOST, mainly. The critical analysis was performed using the recommendations provided by CASPe and compared with the procedure perfomed at the Hospital of the Anexion. Intensive control of glycemia including oral agents, insulin and cardiovascular intervention, glycosylated hemoglobin multiple reflected in less than 7, indicated that there was an evident decrease in the incidence of major macrovascular events such as myocardial infarction, ictus no fatal, and cerebral vascular accident. According to the evidence, maintain glycosylated hemoglobin within normal parameters decreases the risk of macrovascular complications of the disease compared with those patients who drive high glycosylated hemoglobin.

Keywords: complications, diabetes mellitus, glycosylated-hemoglobin

\footnotetext{
${ }^{1}$ Date of reception: November 16, 2011

Date of acceptance: January 16, 2012

${ }^{2}$ Enfermera especialista en Administración de Servicios de Salud. Coordinadora del Programa Hospital de Día. Hospital de la Anexión, Guanacaste.

E. mail: agnesfm2010@hotmail.com

${ }^{3}$ Enfermera Educadora en Servicio. Hospital de la Anexión, Guanacaste. E. mail: silgutio@gmail.com
} 


\section{INTRODUCCION}

La Diabetes Mellitus es una enfermedad que ataca gran cantidad de personas alrededor del mundo y se ha vuelto un problema de Salud Pública, debido a sus complicaciones agudas y crónicas. Analizar el costo humano que representa tanto para el paciente como para su familia, aunado al altísimo costo que representa la atención de estos pacientes para el Sistema de Salud, convierten el tema de la Diabetes Mellitus en punta de lanza para la investigación científica. Jiménez (2000) menciona que "la diabetes mellitus tipo dos afecta del $5 \%$ al $7 \%$ de la población y puede definirse como la punta del témpano de un desorden multifacético metabólico liderado por la hiperglucemia" (p.1)

De acuerdo con Laclé (2008), la diabetes mellitus tipo 2 (DM2) es "una pandemia que afecta tanto a países desarrollados como aquellos en vías de desarrollo" (p.30). El autor afirma que entre los factores que provocan un acelerado crecimiento en el número están el desarrollo económico, el urbanismo y el envejecimiento experimentado por las sociedades en el último siglo, así como cambios en los estilos de vida, especialmente la dieta y la falta de ejercicio, los cuales han originado un aumento de la obesidad, incluso en países pobres (p.30)

Laclé (2008) en los datos publicados en la revista Acta Médica Costarricense menciona que los datos son alarmantes, ya que

La prevalencia mundial de la DM2 en el año 2000 se estimó en $2.8 \%$. Según las proyecciones realizadas habrá un crecimiento global del 46\%para el 2010 y se duplicará en solo 30 años. Esto se traduce en una prevalencia del 4.4\% para un total de 336 millones de diabéticos en el 2030. Este aumento será mayor en los países del tercer mundo, con un incremento del $57 \%$ en Asia, del $50 \%$ en África y del $44 \%$ en América del Sur; porcentajes elevados en comparación con el $23 \%$ proyectado para América del Norte y con el 24\% para Europa (p.30).

Para ampliar todavía más el panorama de Costa Rica, en el mismo documento se rescata la siguiente información:

Costa Rica ha cambiado en las últimas décadas su perfil epidemiológico de morbimortalidad en donde predominan las patologías crónicas de larga evolución y de altos costos, entre las que se destaca la DM2. Esta ocupa el cuarto lugar de las consultas externas y de los egresos hospitalarios, y es la primera causa de los costos hospitalarios. No obstante, no se tienen datos epidemiológicos sobre la prevalencia real de la DM2 de todo el país; sólo se tienen registros indirectos por egresos hospitalarios, prevalencia nacional por autoreferencia y algunos estudios en comunidades o áreas geográficas específicas. El último estudio realizado en el Área Metropolitana en el 2004 estima que la prevalencia de la DM2 es del 7-8 \% de la población mayor de 20 años. De mayor trascendencia es el desconocimiento de la incidencia de la DM2, indicador necesario para la planificación de las estrategias de intervención de esta enfermedad en el país o en una comunidad determinada (p.31). 
Las complicaciones tanto microvasculares como macrovasculares son un tema de abordaje ineludible en el proceso de orientación educativa de nuestros usuarios, máxime teniendo en cuenta que la nefropatía, retinopatía, neuropatía, problemas cardiovasculares y el tratamiento para la prevención de estos daños se ha centrado en nuestro medio en un control intensivo de la glicemia, por lo que el objetivo primordial es sustentar el manejo que se le está dando al paciente Diabético tipo 2, en el que la meta es disminuir los valores de hemoglobina glicosilada hasta parámetros óptimos.

Como menciona González (2011) refiriéndose a un estudio de la DCCT (Diabetes Control and Complications Trials), aunque no se ha encontrado un cierto nivel de HgbAlc que garantice una protección absoluta de las complicaciones tardías, existen varios trabajos que al menos, para algunas de las complicaciones como la Retinopatía y Nefropatía diabética, sugieren que existiría un "nivel crítico" que correspondería a una HgbAlc superior a 8\%, a partir del cual el riesgo sería muy elevado. Lo recomendable es un nivel menor de 7\%.

Según la recomendación dada por Mayo Foundation for Medical Education and Research (MFMER)

La Terapia intensiva de insulina puede prevenir o retrasar la progresión de complicaciones de la diabetes a largo plazo. De hecho, en un estudio, un control estricto de los niveles de azúcar en la sangre reduce el riesgo de ataques cardíacos relacionados con la diabetes y accidentes cerebrovasculares en más del 50 por ciento. (2010)

Estudios previos proporcionan estadísticas atractivas también en las que la Terapia intensiva de insulina puede:

- Reducir el riesgo de daño a los ojos por más de 75 por ciento.

- Reducir el riesgo de daño a los nervios en un 60 por ciento.

- Prevenir o retardar la progresión de la enfermedad renal en un 50 por ciento.

Según la American Diabetes Association (2011) los siguientes son los resultados cuando el HbA1c se está usando para diagnosticar diabetes:

- Normal: menos de $5.7 \%$

- Prediabetes: 5.7 a $6.4 \%$

- Diabetes: $6.5 \%$ o superior

La práctica diaria de la enfermería obliga a las y los profesionales a mantenerse actualizado en estos y otros temas, teniendo siempre en cuenta las necesidades de los usuarios que están bajo sus cuidados, por ello debe manejar herramientas para evaluar la calidad de la información a que tiene acceso, de modo que reciban información con la que conserven su salud y prevengan la enfermedad o bien minimicen el impacto de las complicaciones sobre su calidad de vida.

Dado lo anterior, urge promover la salud y la importancia de desarrollar programas de autocuidado a través de los recursos humanos de enfermería con capacidad técnica para evaluar la información que estos reciben para que puedan transmitir la información. Es en este contexto donde el concepto de la Práctica de la Enfermería Basada en 
la Evidencia toma importancia trascendental porque brinda a las y los profesionales la oportunidad de ser críticos y poder discriminar entre la amplia gama de información que le sea posible accesar, la cual está conformada por cinco etapas:

1. Pregunta derivada del problema.

2. Búsqueda de la información.

3. Evaluación crítica de la información.

4. Implementación de la evidencia en la práctica.

5. Evaluación de la intervención elegida. (Orellana y Paravic, 2007)

Al respecto, Canon (2007), menciona que:

El tema de la práctica de la enfermería basada en la evidencia espolémico, muy actual y de obligado abordaje en el contexto académico, ya que desde el conocimiento de las exigencias de habilitación y acreditaciónde las instituciones prestadoras de servicios de salud (IPS), que imponen el modelo de garantía de la calidad, la elaboración deguías y protocolos basados en los mejores hallazgos disponibles paralas intervenciones de cuidado de la salud de los individuos y colectivos,se requiere que los futuros profesionales de enfermería poseanlas competencias necesarias para investigar y contribuir a brindarcada vez mejores cuidados o para apreciar críticamente la produccióncientífica de nuestros colegas (p.9).

A partir de lo anterior, se plantea entonces la siguiente pregunta clínica de pronóstico en formato PICO:

En pacientes adultos diabéticos tipo 2, ¿El mantenimiento de la hemoglobina glicosilada dentro de parámetros normales, en comparación con aquellos pacientes que manejan hemoglobina glicosilada alta, favorece la disminución del riesgo de sufrir complicaciones macrovasculares de esta patología?

\section{MATERIALES Y METODOS}

En el desarrollo de esta investigación se aplicó la metodología de práctica clínica basada en la evidencia, la cual consta de varias etapas: la primera de ellas correspondió a la formulación de una pregunta de investigación en formato PICO; seguidamente, se implementó una búsqueda de la producción científica sobre el tema que luego fue analizada, de manera que se encuentre una respuesta a la pregunta planteada por las investigadoras.

Para responder a la pregunta de investigación se buscó artículos científicos relacionados con el tema a partir de la base de datos PUBMED y el buscador GOOGLE, herramientas de las que se obtuvieron un total de 551 artículos. Los descriptores utilizados en esta búsqueda fueron los siguientes: Diabetes Mellitus, Type 2[Mesh] AND Hemoglobin A, Glycosylated [Mesh] AND Complications.

Posteriormente, se depuró la información utilizando los siguientes descriptores: hemoglobina glicosilada, diabetes mellitus y complicaciones de la diabetes mellitus. 
Además, se establecieron los siguientes límites: meta-análisis, humanos, estudios de los últimos 5 años, en inglés. Luego de esta depuración se encontraron 6 artículos con los cuales se trabajó para responder a esta pregunta.

Se tomaron en cuenta los artículos que se referían a la relación que existe entre la hemoglobina glicosilada y la prevención de complicaciones para el paciente diabético; dichos artículos fueron sometidos a análisis crítico mediante la aplicación del instrumento CASPe, con el cual se evaluó su calidad. Posteriormente, se determinó que solo un artículo respondía a la cuestión planteada y cumplía con los criterios de calidad.

\section{RESULTADOS}

Se analizó el artículo publicado por el Departamento de Endocrinología del Hospital de Changhai, debido a que este reúne los criterios de calidad del instrumento Caspe, dicho artículo presenta un nivel de confiabilidad del $95 \%$; la población fue seleccionada de forma aleatoria y no se encontraron conflictos de intereses.

A partir de sus resultados, se concluyó que el control intensivo de la glicemia, entre el que se incluyen los agentes orales, la insulina y la intervención cardiovascular múltiple reflejada en hemoglobinas glicosilada menores a 7, indicó que existía una evidente disminución de incidencia de eventos macrovasculares mayores tales como el infarto agudo al miocardio, el ictus no fatal y el accidente vascular cerebral, pero no hubo diferencias significativas en la incidencia de muerte por cualquier otra causa entre el grupo y los controles. (Wu, Xu, Zou, $\mathrm{Han} \& \mathrm{Hu}, 2010)$

Entre los resultados de mayor impacto se puede mencionar que estos estudios demostraron que el grado de control de la glucemia en la DM tipo 2 se relaciona con el riesgo cardiovascular. El análisis de este documento sugiere que por cada subida del $1 \%$ de $\mathrm{HbA1C}$ se produce un aumento del $18 \%$ en el riesgo cardiovascular.

\section{DISCUSION}

Mucho se ha hablado acerca de que la práctica de la Enfermería Basada en la Evidencia no es un concepto innovador, mas sí una herramienta importante para nuestra práctica profesional, ya que brinda a las y los enfermeros lineamientos necesarios para lograr la armonía entre un cuidado enfermero con sustento científico y la sostenibilidad económica del sistema de salud que es punta de lanza para la administración pública. Al respecto, Rojas (2010) menciona que

La práctica clínica basada en la evidencia en una herramienta necesaria, cuya aplicación se justifica por los avances biotecnológicos acelerados en el área de la salud; tal adelanto obliga al profesional de Enfermería a mantenerse informado sobre las mejores prácticas en salud para responder a la población con responsabilidad mediante el desarrollo de una práctica actualizada y fiable que promueva la toma de las mejores decisiones en el quehacer profesional (p.1) 
A partir de lo mencionado, se evidencia que la práctica de la enfermería basada en la evidencia es una destreza por desarrollar en las enfermeras (os), cuyo objetivo es brindar un cuidado con sustento científico que impulse, además, la necesidad de más investigaciones para realizar procesos de trabajo óptimos.

Partiendo de lo dicho, y al plantearse una pregunta clínica como la de esta investigación, se consigue evidencia científica y se validan y actualizan las prácticas de enfermería. La evidencia señaló que, efectivamente, el mantener la hemoglobina glicosilada dentro de parámetros normales disminuye el riesgo de sufrir complicaciones macrovasculares en los pacientes diabéticos en relación con los pacientes que presentan hemoglobina glicosilada alta. Sin embargo, es importante poner especial cuidado al uso de un control intensivo de la glicemia aplicado a la persona adulta mayor, ya que el control metabólico puede influir en el resultado.

Según Plutzky (2011) en su publicación en la American Journal Cardiology expresa que:

El impacto del control glicémico en la reducción de las complicaciones micro vasculares está bien establecida. Aunque más controversial, también hay evidencia de que el control glicémico puede limitar la enfermedad macrovascular, incluyendo CAD, enfermedad arterial periférica y accidente cerebrovascular. Control de la glucemia en el contexto de la diabetes tipo 2, así como la prediabetes, es también entrelazada con factores de riesgo cardiovascular como la obesidad, hipertrigliceridemia y el control de la presión arterial. (traducción propia, p.25b)

Para los profesionales de la salud que laboran en programas para la promoción de la salud es todo un reto realizar proyectos para prevenir complicaciones y estilos de vida que van en detrimento de su condición de salud. No obstante, a partir de los hallazgos de este estudio se vislumbra la necesidad de fortalecer esfuerzos conjuntos del equipo de salud para implementar nuevas estrategias de intervención no farmacológicas, así como el mejoramiento continuo de la calidad de los servicios que promuevan los estilos de vida saludable para el manejo de las personas diabéticas, dado que estos programas son de bajo costo e impactan con efectividad a la población meta (Pérez et al., 2008, p.204)

\section{CONCLUSION}

De acuerdo con la evidencia encontrada, se puede concluir que los pacientes que mantienen la hemoglobina glicosilada dentro de parámetros normales tienen menor riesgo de sufrir complicaciones macrovasculares en comparación con aquellos pacientes que manejan hemoglobina glicosilada alta; sin embargo, se deben tomar en cuenta otros factores de riesgo que puedan favorecer la aparición de estas complicaciones como la obesidad y /o el sedentarismo por mencionar algunos. 


\section{REFERENCIAS}

American Diabetes Association. (2011). Standards of medical care in diabetes Diabetes Care.Jan; 34 Suppl 1:S11-61. Recuperado de: http://care.diabetesjournals.org/content/34/Supplement_1/S11.extract

Canon, H. (2007). Práctica de la enfermería basada en evidencia. Investigación en enfermería, imagen y desarrollo, vol. 9 número 1, pp. 8-18 Pontificia Universidad Javeriana Colombia. Recuperado de: http://redalyc.uaemex.mx/pdf/1452/145212857002.pdf

González, M. (2011) ¿Qué es la hemoglobina glicosilada? Guioteca ¿Qué quieres saber?. Recuperado de: http://www.guioteca.com/diabetes/\%C2\%BFque-es-la-hemoglobina-glicosilada/

Jiménez, M. (2000). Diabetes Mellitus: actualización. Acta Médica Costarricense. Volumen 42. Recuperado desde:http://www.scielo.sa.cr/scielo.php?pid=S0001-

$60022000000200005 \&$ script $=$ sci arttext

Laclé, A., Valero, L. (2008). Incidencia de diabetes tipo 2 en un área urbana marginal de Costa Rica. Acta Médica Costarricense.Volumen 50.Recuperado de: http://www.scielo.sa.cr/scielo.php?pid=S0001$60022008000100006 \&$ script $=$ sci arttext

Mayo Foundation for Medical Education and Research (MFMER).(2010) Intensive insulin therapy: Achieving tight blood sugar control. Recuperado de: http://www.mayoclinic.com/print/intensive-insulintherapy/DA00088/METHOD=print

Orellana, A., Paravic, T. (2007). Enfermería basada en evidencia. Barreras y estrategias para su implementación ciencia y enfermería XIII. Recuperado de: http://www.scielo.cl/scielo.php?pid=S0717$\underline{95532007000100003 \& \text { script }=\text { sci_arttext }}$

Pérez, L., Raigada,J., Collins, A., Alza, S., Parodi, A., Jiménez, S., Casas. J. (2008) Efectividad de un programa educativo en estilos de vida saludables sobre la reducción de sobrepeso y obesidad en el Colegio Robert M. Smith; Huaraz, Ancash, Perú. Acta Med Per 25(4) Recuperado de: http://www.scielo.org.pe/pdf/amp/v25n4/a04v25n4.pdf

Plutzky, J. (2011).Macrovascular Effects and Safety Issues of Therapies for Type 2 Diabetes.Am $J$ Cardiol 2011; 108, (suppl): 25b-32b. Recuperado de: http://www.ajconline.org/article/S00029149(11)01215-X/abstract

Rojas, L. (2012). Curso Bimodal Práctica Clínica de Enfermeria Basada en la Evidencia: Alcances y Limitaciones. Rev. Enfermería Actual en Costa Rica [en línea], No.20 [Recuperado (10 de febrero 2012)]. Disponible World Wide Web: <http://www.revenf.ucr.ac.cr/ ebe.pdf> ISSN 1409-4568 


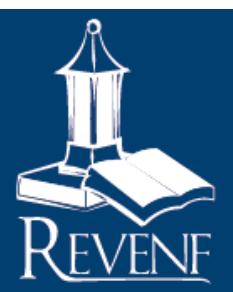

Wu, H., Xu, MJ., Zou, DJ., Han, QJ., Hu, X. (2010). Intensive glycemic control and macrovascular events in type 2 diabetes mellitus: a meta-analysis of randomized controlled trials. Department of Endocrinology, Changhai Hospital, Second Military Medical University, Shanghai, China. Recuperado de: http://www.ncbi.nlm.nih.gov/pubmed/21034605. 